\title{
Guest Editors' Introduction: special issue of selected papers from ECML PKDD 2011
}

\author{
Dimitrios Gunopulos • Donato Malerba • \\ Michalis Vazirgiannis
}

Received: 12 June 2012 / Accepted: 19 June 2012 / Published online: 5 July 2012

(C) The Author(s) 2012

The last edition of the European Conference on Machine Learning and Principles and Practice of Knowledge Discovery in Databases (ECML PKDD) was held in Athens, Greece, during September 5-9, 2011. Ten years after the first edition of this joint conference, ECML PKDD 2011 continued to provide a common forum for the closely related fields of machine learning and data mining. Apart from six plenary invited talks, four invited talks for the industrial session, a demo session, six tutorials and eleven co-located workshops, the main technical sessions comprised the presentation of 121 peer-reviewed papers selected by the program committee from 599 full-paper submissions. ECML PKDD 2011 was a highly selective conference and the proceedings were published in three volumes of the Springer's Lecture Notes in Artificial Intelligence series (Gunopulos et al. 2011a,b,c).

In the previous three editions of the conference, the best selected papers on data mining were published in a special issue of this journal in advance of the conference and were not included in the conference proceedings. A novelty introduced in ECML PKDD 2011 is the post-conference publication of the special issue in order to guarantee the high-standard reviews expected for this top quality journal. Therefore, authors of the best ten papers in data mining have been invited to submit a

\footnotetext{
D. Gunopulos

University of Athens, Panepistimiopolis, 15784 Athens, Greece

e-mail: dg@di.uoa.gr

D. Malerba $(\varangle)$

Dipartimento di Informatica, Università degli Studi di Bari "Aldo Moro",

via Orabona 4, 70125 Bari, Italy

e-mail: donato.malerba@uniba.it

M. Vazirgiannis

Athens University of Economics \& Business, 12 Kodrigktonos, 11257 Athens, Greece

e-mail: mvazirg@aueb.gr
} 
significantly extended version of their paper to this special issue. The selection was made by Program Chairs on the basis of their exceptional scientific quality and high impact on the field, as indicated by conference reviewers.

In this special issue you will find eight papers which have been accepted after two or three rounds of peer-reviewing according to the journal criteria. The diversity of topics addressed in these papers reflects the significant progress being made by the data mining community in the theoretical understanding of the principles underlying knowledge discovery in databases, as well as in the application of tools and techniques to real-world problems. We believe these works have the potential to spur new research in the field.

The paper "Comparing Apples and Oranges: Measuring Differences between Exploratory Data Mining Results" (Tatti and Vreeken 2012), provides us a means to highlight the differences between the results of heterogeneous exploratory data mining methods. The rather general question "what can you tell me about my data?" can be answered by different data mining methods, which do not share a common formal goal and hence generates results that are not easily comparable. To face this problem, the authors propose to transform the results of different exploratory data mining methods into a common language that help us to measure the informativeness of the results and their overlap. The proposed method is for results obtained on binary data, but extensions to richer data types are anticipated.

The paper "Diverse Subgroup Set Discovery" (van Leeuwen and Knobbe 2012), advances the state of the art in subgroup discovery, that is the discovery of subsets of the data, where the target attributes show an interesting difference in distribution, compared to that of the entire dataset. The notion of "diversity" in sets of subgroups is introduced here to face two important problems relating to subgroup discovery from large datasets: the high levels of redundancy occurring in the final pattern set and the computational inefficiency of complete solutions discovery. The authors propose to discover diverse subgroups sets rather than just subgroups, showing that this way it is possible to balance subgroup quality, diversity and runtime.

The paper "Finding Density-Based Subspace Clusters in Graphs with Feature Vectors" (Günnemann et al. 2012), focuses on clustering networked data, where both relationships between objects as well as features of objects are considered to extract meaningful clusters. Dense subgraph mining, which aims to cluster graph nodes on the basis of their connectivity, and subspace clustering, which aims to cluster objects on the basis of their attribute-value similarity, are here combined in a novel iterative algorithm to detect clusters in networked data. Analytical proofs of the correctness, convergence, and complexity of the algorithm complement a careful experimental evaluation.

The paper “Actively Learning to Infer Social Ties" (Zhuang et al. 2012), investigates how to predict social relationships between people in a large online social network. This problem is of practical importance for many emerging applications, but at the same time it poses several challenges, such as how to exploit the few labelled relationships, or how to make optimal use of user interaction. In this paper, it is faced by using a factor graph model which takes into account the features of the edge, as well as the correlations and constraints between different edges. Parameters of the model are estimated so that the log-likelihood of labeled relationships is maximized. Two 
strategies are also proposed to actively select potentially wrong but most useful relationships to query the user. Factor graph model partitioning and parallel computation make this method applicable to large online social networks.

The paper "Tensor Factorization Using Auxiliary Information" (Narita et al. 2012), is concerned with tensors (or multi-way arrays) which are a suitable representation for multi-object relationships. Tensor analysis aims at investigating the relations which remain valid when we change from one coordinate system to another. This paper focus on tensor completion, a specific tensor analysis task, which aims at imputing missing values of an incomplete tensor. The state-of-the-art methods for the prediction of missing values are inaccurate when observations are sparse. The idea presented here is that of incorporating external knowledge in tensor decomposition, thus ultimately improving the accuracy of tensor completion methods. Also in this case, large collections of social data offer the right context where this idea could be profitably applied.

The paper "Fast Support Vector Machines for Convolution Tree Kernels" (Severyn and Moschitti 2012), addresses the scalability issue for kernel methods applied to treestructured data. The idea is that of compactly representing trees using DAGs in order to avoid redundant kernel evaluations. An abstract efficiency measure is defined in order to verify the speedup of the proposed algorithm independently of the hardware used in the experiments. The main core of this work is complemented by both a parallelization approach and a method for handling imbalanced datasets, which enlarge the range of potential applications to real-world problems.

The paper "Fast projections onto mixed-norm balls with applications" (Sra 2012), brings forward fast methods for computing gradient projections in regularized learning settings where the regularizer is in the form of an $l_{p, q}$-mixed-norm. This mixed-norm regularization is attractive in many applications of regression and classification in that it facilitates group sparsity in the model. Nevertheless, the resulting optimization problem is challenging to solve. This paper faces the problem by considering a constrained formulation and motivates this choice by presenting some advantages over the penalized formulation.

The paper "Network Regression with Predictive Clustering Trees" (Stojanova et al. 2012), proposes a novel method for learning regression models from network data, which are typically characterized by positive autocorrelation. Although the autocorrelation reveals the violation of the common i.i.d. assumption of training data made by many learning systems, it also offers a unique opportunity to improve the performance of the learned models, since inferences about one node in the network can be used to improve inferences of related entities. This opportunity is taken in the method proposed here, since autocorrelation is considered when building regression models in the form of predictive clustering trees, i.e. generalizations of decision trees that can be used for clustering, prediction and multi-target prediction.

We hope the readers will enjoy reading these articles and will find in them a source of inspiration for their work.

Acknowledgments We wish to warmly thank all authors who submitted their papers to this special issue. We are indebted to the reviewers for their careful work and for their constructive comments to the authors. We are grateful to the journal Editor-in-Chief Dr. Geoff Webb for his great help and support in organizing this special issue. A special thank to Gayathri Balasubramanian from the Springer editorial staff for her tireless support and troubleshooting in the production of this special issue. 


\section{References}

Günnemann X, Boden B, Seidl T (2012) Finding density-based subspace clusters in graphs with feature vectors. Data Min Knowl Discov 25(2). doi:10.1007/s10618-012-0272-z

Gunopulos D, Hofmann T, Malerba D, Vazirgiannis M (2011a) Machine learning and knowledge discovery in databases-European conference, ECML PKDD 2011, Proceedings, Part I, Athens, Greece, September 5-9, 2011. Lecture Notes in Computer Science, vol 6911. Springer

Gunopulos D, Hofmann T, Malerba D, Vazirgiannis M (2011b) Machine learning and knowledge discovery in databases-European conference, ECML PKDD 2011, Proceedings, Part II, Athens, Greece, September 5-9, 2011. Lecture Notes in Computer Science, vol 6912. Springer

Gunopulos D, Hofmann T, Malerba D, Vazirgiannis M (2011c) Machine learning and knowledge discovery in databases-European conference, ECML PKDD 2011, Proceedings, Part III, Athens, Greece, September 5-9, 2011. Lecture Notes in Computer Science, vol 6913. Springer

Narita A, Hayashi K, Tomioka R, Kashima H (2012) Tensor factorization using auxiliary information. Data Min Knowl Discov 25(2). doi:10.1007/s10618-012-0280-z

Severyn A, Moschitti A (2012) Fast support vector machines for convolution tree kernels. Data Min Knowl Discov 25(2). doi:10.1007/s10618-012-0276-8

Sra S (2012) Fast projections onto mixed-norm balls with applications. Data Min Knowl Discov 25(2). doi:10.1007/s10618-012-0277-7

Stojanova D, Ceci M, Appice A, Džeroski S (2012) Network regression with predictive clustering trees. Data Min Knowl Discov 25(2). doi:10.1007/s10618-012-0278-6

Tatti N, Vreeken J (2012) Comparing apples and oranges: measuring differences between exploratory data mining results. Data Min Knowl Discov 25(2). doi:10.1007/s10618-012-0275-9

van Leeuwen M, Knobbe A (2012) Diverse subgroup set discovery. Data Min Knowl Discov 25(2). doi:10.1007/s10618-012-0273-y

Zhuang H, Tang J, Tang W, Lou T, Chin A, Wang X (2012) Actively learning to infer social ties. Data Min Knowl Discov 25(2). doi:10.1007/s10618-012-0274-X 